\title{
On classical and quantum $q$-oscillators in the relativistic theory ${ }^{*}$ )
}

\author{
R.M. Mir-Kasimov \\ Bogoliubov Laboratory of Theoretical Physics, Joint Institute for Nuclear Research \\ 141980, Dubna, Russia \\ and \\ Department of Mathematics, Izmir Institute of Technology, 35430, Urla/Izmir, Turkey
}

Received 4 September 2004

The factorization method, applied to the finite-difference Schrödinger equation in the relativistic configurational space, allows to consider the $q$-deformations as a relativistic effect. In particular, different factorizations allow to obtain all known $q$-oscillators in a unified way. The classical limit of deformed Hamiltonians is investigated.

PACS: 02.20.Uw, 03.30.+p

Key words: quantum, classical, relativistic, oscillator

In [1] the relativistic oscillator was considered on the basis of the concept of the relativistic configurational representation $[2,3]$. Referring the reader to $[1,4,5]$, we present here the necessary formulae (the one-dimensional case is considered).

The relativistic finite-difference Schrödinger equation (we use the unit system $\hbar=m=c$ if not specified otherwise)

$$
\left(\hat{h}_{0}+V(x)-e\right) \psi(x)=\left(\frac{\hat{k}^{2}}{2 m}+V(x)-\frac{k^{2}}{2 m}\right) \psi(x)=0,
$$

where the relativistic energy-momentum vector $\left(p^{0}=\cosh \chi, p=\sinh \chi\right)$ is connected with the kinetic momentum $k$ and energy $e$ :

$$
h=\frac{\hat{k}^{2}}{2}+V(x), \quad e=\frac{k^{2}}{2}, \quad \hat{k}=-2 m c \sinh \left(\frac{\mathrm{i} \hbar}{2 m c} \frac{\mathrm{d}}{\mathrm{d} x}\right), \quad k=2 m c \sinh \frac{\chi}{2} .
$$

Parameter $\chi$ is the hyperbolic angle (the rapidity) (see $[1,4,5]$ ).

In [1] the general approach to the oscillators in the relativistic-configurational space were formulated and one of such oscillators was considered in detail. It was shown that the relativistic oscillator is the $q$-oscillator with parameter of deformation depending on physical parameters

$$
q=\exp \left(-\frac{\omega \hbar}{4 m c^{2}}\right)
$$

\footnotetext{
*) Presented at the $13^{\text {th }}$ International Colloquium on "Integrable Systems and Quantum Groups", Prague, 17-19 June 2004.
} 
The ladder operators $A^{ \pm}$(we use in what follows the unit system $\hbar=c=m=1$ )

$$
\begin{aligned}
& A^{ \pm}= \pm \mathrm{i} \sqrt{2} \mathrm{e}^{ \pm \omega / 8} {\left[\sinh \left(\frac{\mathrm{i}}{2} \frac{\mathrm{d}}{\mathrm{d} x}\right) \mp \mathrm{i} \tan \frac{\omega x}{2} \cosh \left(\frac{\mathrm{i}}{2} \frac{\mathrm{d}}{\mathrm{d} x}\right)\right]=} \\
&=\mp \frac{1}{\sqrt{2}} \mathrm{e}^{ \pm \omega x^{2} / 2} \widehat{\mathcal{D}} \mathrm{e}^{-\omega x^{2} / 2}
\end{aligned}
$$

where

$$
\widehat{\mathcal{D}}=-\frac{2 \mathrm{i}}{\cos \left(\frac{1}{2} \omega x\right)} \cdot \sinh \left(\frac{\mathrm{i}}{2} \frac{\mathrm{d}}{\mathrm{d} x}\right)
$$

obey the $q$-mutation relations $[1],[7-9]$

$$
\left[A^{-}, A^{+}\right]_{q}=q A^{-} A^{+}-q^{-1} A^{+} A^{-}=2\left(q^{-1}-q\right)=4 \sinh \frac{1}{4} \omega .
$$

The systematic analysis, see below (7)-(9), shows, however, that unlike the standard differential non-relativistic Schrödinger equation, which can be factorized in only one way (for a given potential), for the relativistic finite-difference Schrödinger equation, there are a number of different factorizations. Each of them leads in general to a specific $q$-oscillator model. We show that there are different possibilities to obtain factorized oscillator-like relativistic Hamiltonian. The origin of this fact lies in the finite-difference character of the relativistic momentum operator (2). We can obtain also different finite-difference ladder operators. Indeed, let us return for a moment to the non-relativistic QM. The free-energy operator is the second derivative, which can be split in two factors by only one way, if we do not consider the fractional degrees of differentiation operators:

$$
H_{0}=\frac{p^{2}}{2 m}=-\frac{1}{2 m} \frac{\mathrm{d}^{2}}{\mathrm{~d} x^{2}}=-\frac{1}{2 m}\left(\frac{\mathrm{d}}{\mathrm{d} x}\right)\left(\frac{\mathrm{d}}{\mathrm{d} x}\right) .
$$

In the relativistic case the free Hamiltonian is the finite-difference operator, given by (2). Using the definition of the hyperbolic function $\sinh z$ :

$$
\sinh \left(\frac{\mathrm{i}}{2} \frac{\mathrm{d}}{\mathrm{d} x}\right)=\frac{1}{2}\left[\exp \left(\frac{\mathrm{i}}{2} \frac{\mathrm{d}}{\mathrm{d} x}\right)-\exp \left(-\frac{\mathrm{i}}{2} \frac{\mathrm{d}}{\mathrm{d} x}\right)\right]
$$

and the relation $\exp \left( \pm \frac{1}{2} \mathrm{i} d / \mathrm{d} x\right)\langle x \mid p\rangle=\mathrm{e}^{\mp \chi / 2}\langle x \mid p\rangle$, we can write the free Schrödinger equation in different forms:

$$
\begin{gathered}
{\left[1-\exp \left(-\mathrm{i} \frac{\mathrm{d}}{\mathrm{d} x}\right)^{2}\right]\langle x \mid p\rangle=2 \mathrm{e}^{\chi} \sinh ^{2}\left(\frac{1}{2} \chi\right)\langle x \mid p\rangle,} \\
{\left[1-\exp \left(\mathrm{i} \frac{\mathrm{d}}{\mathrm{d} x}\right)^{2}\right]\langle x \mid p\rangle=2 \mathrm{e}^{-\chi} \sinh ^{2}\left(\frac{1}{2} \chi\right)\langle x \mid p\rangle,} \\
{\left[1-\exp \left(\mathrm{i} \frac{\mathrm{d}}{\mathrm{d} x}\right)\right]\left[1-\exp \left(-\mathrm{i} \frac{\mathrm{d}}{\mathrm{d} x}\right)\right]\langle x \mid p\rangle=-2 \sinh ^{2}\left(\frac{1}{2} \chi\right)\langle x \mid p\rangle .}
\end{gathered}
$$


From (7)-(9) different $q$-oscillators can be obtained. Referring the reader to $[1,4,5]$, we write down here the corresponding ladder operators. For the case (7) we have the ladder operators

$$
\begin{aligned}
& \eta^{+}=-\frac{\mathrm{i}}{\sqrt{2}} \mathrm{e}^{\mathrm{i} \omega x(\xi-2)}\left[1-\exp \left(\mathrm{i} \frac{\omega x}{2}\right) \exp \left(-\mathrm{i} \frac{\mathrm{d}}{\mathrm{d} x}\right) \exp \left(\mathrm{i} \frac{\omega x}{2}\right)\right] \mathrm{e}^{\mathrm{i} \omega x \xi}, \\
& \eta^{-}=\frac{\mathrm{i}}{\sqrt{2}} \mathrm{e}^{\mathrm{i} \omega x(\xi+2) \xi}\left[1-\exp \left(-\mathrm{i} \frac{\omega x}{2}\right) \exp \left(-\mathrm{i} \frac{\mathrm{d}}{\mathrm{d} x}\right) \exp \left(-\mathrm{i} \frac{\omega x}{2}\right)\right] \mathrm{e}^{-\mathrm{i} \omega x \xi} .
\end{aligned}
$$

In this case the value of $q$ is

$$
\tilde{q}=e^{\omega}
$$

The $q$-mutator is

$$
\left[\eta^{-}, \eta^{+}\right]_{\tilde{q}}=\tilde{q} \eta^{-} \eta^{+}-\tilde{q}^{-1} \eta^{+} \eta^{-}=\mathrm{e}^{\omega} \eta^{-} \eta^{+}-\mathrm{e}^{-\omega} \eta^{+} \eta^{-}=\frac{1}{2}\left(\tilde{q}-\tilde{q}^{-1}\right)=\sinh \omega
$$

Another pair of the creation and annihilation operators $\gamma^{ \pm}$comes out if we start with the free Hamiltonian from (9)

$$
\begin{aligned}
\gamma^{+}=\frac{\mathrm{i}}{\sqrt{2}} \exp \left(-\mathrm{i}(2+\zeta) \frac{\mathrm{d}}{\mathrm{d} x}\right) & {\left[1-\exp \left(-\mathrm{i} \frac{\omega x}{2}\right) \exp \left(\mathrm{i} \frac{\mathrm{d}}{\mathrm{d} x}\right) \mathrm{e}\left(-\mathrm{i} \frac{\omega x}{2}\right)\right] } \\
& \times \exp \left(\mathrm{i} \zeta \frac{\mathrm{d}}{\mathrm{d} x}\right), \\
\gamma^{-}=\frac{\mathrm{i}}{\sqrt{2}} \exp \left(\mathrm{i} \zeta \frac{\mathrm{d}}{\mathrm{d} x}\right)[1-\exp & \left.\left(-\mathrm{i} \frac{\omega x}{2}\right) \exp \left(-\mathrm{i} \frac{\mathrm{d}}{\mathrm{d} x}\right) \exp \left(-\mathrm{i} \frac{\omega x}{2}\right)\right] \\
& \times \exp \left(\mathrm{i}(2-\zeta) \frac{\mathrm{d}}{\mathrm{d} x}\right) .
\end{aligned}
$$

The value of $q$ is

$$
\tilde{\tilde{q}}=\tilde{q}^{-1}=\mathrm{e}^{-\omega}
$$

and the $q$-mutator is

$$
\left[\gamma^{-}, \gamma^{+}\right]_{\tilde{q}^{-1}}=\tilde{q}^{-1} \gamma^{-} \gamma^{+}-\tilde{q} \gamma^{+} \gamma^{-}=\mathrm{e}^{-\omega} \gamma^{-} \gamma^{+}-\mathrm{e}^{\omega} \gamma^{+} \gamma^{-}=\frac{1}{2}\left(\tilde{q}-\tilde{q}^{-1}\right)
$$

The pairs of the ladder operators $\eta^{ \pm}$and $\gamma^{+}$are symmetric in the sense that they transform into each other if we exchange the coordinates and rapidities. This generalizes the symmetry between coordinates and momenta existing in the nonrelativistic theory. Now let us consider the classical limit of this relativistic theory.

We concentrate on the case described by the ladder operators (4). Using BakerCampbell-Hausdorf formula, we can rewrite $A^{+}, A^{-}$operators as

$$
\begin{aligned}
A^{ \pm} & =\mp \frac{\sqrt{2}}{\cos (\omega x / 2)} \mathrm{e}^{ \pm \omega x^{2} / 2} \sinh \left(\frac{\mathrm{i}}{2} \frac{\mathrm{d}}{\mathrm{d} x}\right) \mathrm{e}^{\mp \omega x^{2} / 2}= \\
& =\frac{\sqrt{2}}{\cos (\omega x / 2)} \sinh \left(\frac{p+\mathrm{i} \omega x}{2}\right)=\frac{\sqrt{2}}{\cos (\omega x / 2)} \sinh \left(\frac{a^{ \pm}}{\sqrt{2}}\right),
\end{aligned}
$$


where

$$
a^{ \pm}=\frac{1}{\sqrt{2}}(p \pm \mathrm{i} \omega x)
$$

are standard non-relativistic ladder operators.

Note that in the classical limit the deformation parameter

$$
q=\exp \left(-\frac{\omega \hbar}{4 m c^{2}}\right) \longrightarrow 1
$$

Correspondingly the "ladder functions" (12) go over to the classical operators

$$
\begin{aligned}
& B^{+}=\sqrt{2 m c^{2}}\left(\sinh \frac{p}{2 m c}+\mathrm{i} \tan \frac{\omega x}{2 c} \cosh \frac{p}{2 m c}\right), \\
& B^{-}=\sqrt{2 m c^{2}}\left(\sinh \frac{p}{2 m c}-\mathrm{i} \tan \frac{\omega x}{2 c} \cosh \frac{p}{2 m c}\right) .
\end{aligned}
$$

The relativistic classical Hamiltonian function is defined as

$$
H=B^{+} B^{-}=2 m c^{2}\left(\cosh ^{2} \frac{p}{2 m c} \cos ^{-2} \frac{\omega x}{2 c}-1\right) .
$$

The Poisson bracket for $B^{+}$and $B^{-}$is

$$
\begin{aligned}
\left\{B^{-}, B^{+}\right\} & =\frac{\partial B^{-}}{\partial x} \frac{\partial B^{+}}{\partial p}-\frac{\partial B^{-}}{\partial p} \frac{\partial B^{+}}{\partial x}=-\mathrm{i} \omega \cosh ^{2} \frac{p}{2 m c} \cos ^{-2} \frac{\omega x}{2 c}= \\
& =-\mathrm{i} \omega\left[\frac{H}{2 m c^{2}}+1\right] \stackrel{c \rightarrow \infty}{\longrightarrow}-\mathrm{i} \omega,
\end{aligned}
$$

which is a right non-relativistic limit

$$
\left\{a^{-}, a^{+}\right\}=-\mathrm{i} \omega .
$$

Correspondingly the equations of motion have the form

$$
\begin{gathered}
\frac{\mathrm{d} B^{+}}{\mathrm{d} t}=\left\{B^{-}, H\right\}=\left\{B^{-}, B^{-} B^{+}\right\}=\left\{B^{+}, B^{-}\right\} B^{+}=\mathrm{i} \omega\left[\frac{H}{2 m c^{2}}+1\right] B^{+}, \\
\frac{\mathrm{d} B^{-}}{\mathrm{d} t}=-\mathrm{i} \omega\left[\frac{H}{2 m c^{2}}+1\right] B^{-} .
\end{gathered}
$$

Direct calculation gives

$$
\begin{aligned}
\frac{\mathrm{d} H}{\mathrm{~d} t}= & \frac{\mathrm{d}}{\mathrm{d} t}\left(B^{+} B^{-}\right)= \\
= & 2 m c \frac{\cosh (p /(2 m c))}{\cos ^{2}(\omega x /(2 c))}\left[\frac{\dot{p}}{m} \sinh \frac{p}{2 m c}+\omega \dot{x} \tan \frac{\omega x}{2 c} \cosh \frac{p}{2 m c}\right]= \\
& \stackrel{c \rightarrow \infty}{\longrightarrow} p\left(\frac{\dot{p}}{m}+\omega^{2} x\right)=p\left(\frac{\ddot{p}}{m}+\omega^{2} x\right)= \\
= & 0 \quad \text { for the non-relativistic oscillator. }
\end{aligned}
$$


But what is the value of $\mathrm{d} H / \mathrm{d} t$ in the relativistic case? (0?)

Let us introduce, by analogy with non-relativistic relations, the new relativistic "coordinate" $\kappa$ and "momentum" $\pi$ :

$$
\begin{aligned}
& \kappa=\frac{\mathrm{i}}{\omega \sqrt{2 m}}\left(B^{-} B^{+}\right)=\frac{2 c}{\omega} \tan \frac{\omega x}{2 c} \cosh \frac{p}{2 m c}, \\
& \pi=\sqrt{\frac{m}{2}}\left(B^{+}+B^{-}\right)=2 m c \sinh \frac{p}{2 m c} .
\end{aligned}
$$

In terms of these relativistic coordinate and momentum the Hamiltonian takes the form

$$
H=\frac{\pi^{2}}{2 m}+\frac{m \omega^{2} \kappa^{2}}{2} .
$$

The Hamiltonian equations of motion for $\kappa$ and $\pi$ take the form

$$
\begin{aligned}
& \dot{\kappa}=\{\kappa, H\}=\left(\frac{H}{2 m c^{2}}+1\right) \frac{\pi}{m}, \\
& \dot{\pi}=\{\kappa, H\}=-\omega^{2} m\left(\frac{H}{2 m c^{2}}+1\right) \kappa .
\end{aligned}
$$

As $H$ does not explicitly depend on time, the following equation of motion is satisfied:

$$
\ddot{\kappa}+\omega^{2}\left(\frac{H}{2 m c^{2}}+1\right)^{2} \kappa=0 .
$$

Further using

$$
\{\kappa, H\}=\frac{\partial \kappa}{\partial x} \frac{\partial H}{\partial p}-\frac{\partial \kappa}{\partial p} \frac{\partial H}{\partial x}
$$

and

$$
\{\pi, H\}=-\frac{\partial \pi}{\partial p} \frac{\partial H}{\partial x},
$$

we explicitly calculate the Poisson brackets in (24) and (25). Then we calculate as well the time derivatives of the usual coordinate and momentum, expressing them in terms of relativistic quantities

$$
\begin{aligned}
\dot{x} & =\{x, H\}=\frac{\partial H}{\partial p}=2 c \sinh \frac{p}{2 m c} \cosh \frac{p}{2 m c} \cos ^{-2} \frac{\omega x}{2}= \\
& =\left[\frac{H}{2 m c^{2}}+1\right] \cosh ^{-1} \frac{p}{2 m c} \frac{\pi}{m}, \\
\dot{p} & =\{p, H\}=-\frac{\partial H}{\partial x}=-2 m c \omega \tan \frac{\omega x}{2 c} \cosh ^{2} \frac{p}{2 m c} \cos ^{-2} \frac{\omega x}{2 c}= \\
& =-m \omega^{2}\left[\frac{H}{2 m c^{2}}+1\right] \cosh ^{-1} \frac{p}{2 m c} \kappa .
\end{aligned}
$$


Inserting these results into the expression (21) for the time derivative of the Hamiltonian $\mathrm{d} H / \mathrm{d} t$ obtained by direct differentiation, we come to the important conclusion that

$$
\frac{\mathrm{d} H}{\mathrm{~d} t} \equiv 0 .
$$

In other words the Hamiltonian equations for $x$ and $p$, and for $\kappa$ and $\pi$ are compatible. The reason of that fact lies in the periodicity properties [6] of the relativistic classical oscillator Hamiltonian function (16):

$$
H(x, p+2 \pi \mathrm{i} m c)=H(x, p)
$$

and

$$
H\left(x+\frac{2 \pi c}{\omega}, p\right)=H(x, p) .
$$

As a consequence of Eqs. (32) and (33) the trajectories in $(\kappa, \pi)$-phase space have the double periodic structure. The orbits, corresponding to the constant energy in $(\kappa, \pi)$-phase space (cf. 23$)$, are multiply reproduced in the $(x, p)$-phase space exhibiting the periodic dependence. Of course the scales along axes in these phase spaces are different. To see the manifestation of the periodicity property described by $(32)$, we have to consider the complex $(x, p)$-phase space.

\section{References}

[1] R.M. Mir-Kasimov: J. Phys. A. 24 (1991) 4283.

[2] V.G. Kadyshevsky, R.M. Mir-Kasimov, and N.B. Skachkov: Nuovo Cimmento 55 (1968) 233.

[3] R.M. Mir-Kasimov: Foundations Phys. 32 (2002) 607.

[4] R.M. Mir-Kasimov and G. Tanoglu: in Selected Problems of Modern Physics (Eds. B.M. Barbashov et al.), Joint Institute for Nuclear Research, Dubna, 2003, p. 148.

[5] R.M. Mir-Kasimov, G. Tanoglu, and S. Atilgan: in Supersymetries and Quantum Symmetries (Eds. E.A. Ivanov and A.I. Pashnev), Joint Institute for Nuclear Research, Dubna, 2004, p. 416.

[6] R.M. Mir-Kasimov, K. Koizumi, and I.S. Sogami: Progr. Theor. Phys. 110 (2003) 819.

[7] M. Arik and D.D. Coon: J. Math. Phys. 17 (1975) 524.

[8] A.J. Macfarlane: J. Phys. A 22 (1989) 4581.

[9] L.C. Biedenharn: J. Phys. A 22 (1989) L873. 